Research Paper

\title{
Maternal Active Mastication during Prenatal Stress Ameliorates Prenatal Stress-Induced Lower Bone Mass in Adult Mouse Offspring
}

Kagaku Azuma ${ }^{\circledR}$, Minori Ogura², Hiroko Kondo², Ayumi Suzuki², Sakurako Hayashi², Mitsuo linuma², Minoru Onozuka 3 , Kin-ya Kubo ${ }^{4}$

1. Department of Anatomy, School of Medicine, University of Occupational and Environmental Health, 1-1 Iseigaoka, Yahatanishi-ku, Kitakyushu, Fukuoka, 807-8555, Japan;

2. Department of Pediatric Dentistry, Asahi University School of Dentistry, 1851 Hozumi, Mizuho, Gifu, 501-0296, Japan;

3. Department of Judo Therapy and Medical Science, Faculty of Medical Science, Nippon Sport Science University, Yokohama Kanagawa, 227-0033, Japan;

4. Seijoh University Graduate School of Health Care Studies, 2-172, Fukinodai, Tokai, Aichi, 476-8588, Japan.

$\triangle$ Corresponding author: Dr. Kagaku Azuma MD. PhD., Department of Anatomy, School of Medicine, University of Occupational and Environmental Health, 1-1 Iseigaoka, Yahatanishi-ku, Kitakyushu, Fukuoka, 807-8555, Japan. Telephone number: +8193691 7418; Fax number: +81 93 691 8544 E-mail address:kazuma@med.uoeh-u.ac.jp

(C) Ivyspring International Publisher. This is an open access article distributed under the terms of the Creative Commons Attribution (CC BY-NC) license (https://creativecommons.org/licenses/by-nc/4.0/). See http://ivyspring.com/terms for full terms and conditions.

Received: 2016.11.10; Accepted: 2017.01.31; Published: 2017.04.06

\begin{abstract}
Chronic psychological stress is a risk factor for osteoporosis. Maternal active mastication during prenatal stress attenuates stress response. The aim of this study is to test the hypothesis that maternal active mastication influences the effect of prenatal stress on bone mass and bone microstructure in adult offspring. Pregnant ddY mice were randomly divided into control, stress, and stress/chewing groups. Mice in the stress and stress/chewing groups were placed in a ventilated restraint tube for 45 minutes, 3 times a day, and was initiated on day 12 of gestation and continued until delivery. Mice in the stress/chewing group were allowed to chew a wooden stick during the restraint stress period. The bone response of 5-month-old male offspring was evaluated using quantitative micro-CT, bone histomorphometry, and biochemical markers. Prenatal stress resulted in significant decrease of trabecular bone mass in both vertebra and distal femur of the offspring. Maternal active mastication during prenatal stress attenuated the reduced bone formation and increased bone resorption, improved the lower trabecular bone volume and bone microstructural deterioration induced by prenatal stress in the offspring. These findings indicate that maternal active mastication during prenatal stress can ameliorate prenatal stress-induced lower bone mass of the vertebra and femur in adult offspring. Active mastication during prenatal stress in dams could be an effective coping strategy to prevent lower bone mass in their offspring.
\end{abstract}

Key words: Bone histomorphometry, Chewing, Micro-CT, Osteoporosis, Prenatal stress

\section{Introduction}

Osteoporosis is the most common skeletal disorder characterized by low bone mass and deterioration of bone quality, leading to an increased risk of fragility fracture $[1,2]$. Osteoporosis is a major cause of mortality and morbidity through its association with age-related fractures. The vertebra and proximal femur are highly susceptible to fractures in osteoporotic patients [1-3]. With the rapid increase in the elderly population, the number of osteoporotic fractures worldwide is on the rise, which will pose increasingly important health and socioeconomic burden. Therefore, there is an urgent need to direct at identifying effective strategies for preventing osteoporosis.

Osteoporosis has a complex pathogenesis and multifactorial etiology, including genetic and environmental components. Several factors, such as menopause, physical inactivity, cigarette smoking, 
and alcohol abuse have been implicated in the development of osteoporosis [4]. Chronic psychological stress is a major health concern, as it is a risk factor for various diseases, including cancer, cardiovascular disorder, obesity, diabetes, and neurodegenerative diseases $[5,6]$. Recently, the relationship between chronic psychological stress and osteoporosis has been the subject of a growing body of research. Animal studies with chronic psychological stress showed a significant decrease of bone mass and deterioration of bone quantity [7-11].

Osteoporosis is one of many chronic conditions that might be influenced by the entire life course including early life events during pregnancy [12-14]. Bone size and bone density increases from embryogenesis through intrauterine, infant, childhood, and adolescence to reach a peak in early adulthood. The peak bone mass achieved is a strong predictor of later osteoporosis risk. The concept that poor nutrition during organ development could lead to increased risk of disease later in life was originally called the Barker hypothesis, then the fetal origin of adult disease, and now the developmental origins of health and disease [15]. A substantial body of evidence indicates that early life events, including maternal nutritional state and maternal stress, influence the development of many diseases, such as diabetes, obesity, cardiovascular diseases, osteoporosis, and neurodegenerative diseases [15, 16].

Active mastication, or chewing, plays an important role in maintaining mental, physical, and physiological functions. Chewing is also a practical stress-coping behavior [12, 17-19]. Our recent studies showed that chewing under chronic stress could ameliorate stress-induced lower bone mass by attenuating the reduced bone formation and increased bone resorption [9-11]. Chewing may help to preserve the bone strength by suppressing the hypothalamic-pituitary-adrenocortical (HPA) axis [9-12, 17-19]. Maternal chewing during prenatal stress attenuated the increased corticosterone level induced by prenatal stress. In the present study we examine whether maternal chewing during prenatal stress ameliorates stress-induced lower trabecular bone and bone microstructural deterioration in adult offspring of mice.

\section{Materials and Methods}

\section{Animals and experimental procedures}

ddY mice were purchased from Chubu Kagaku Shizai Co., Ltd. (Nagoya, Japan) and housed in the Division of Animal Experiments, Asahi University School of Dentistry under temperature- and humidity-controlled conditions $\left(23 \pm 1^{\circ} \mathrm{C}, 55 \pm 25 \%\right)$ and a 12:12 h light/dark cycle (light period, 6:00-18:00; dark period, 18:00-6:00). All animals had free access to tap water and standard rodent pellet chow (CE-2, CLEA Japan, Inc., Tokyo, Japan). All experiments were performed according to the guidelines for the care and use of laboratory animals of Asahi University. The ethics committee of Asahi University School of Dentistry approved the study. Twelve-week-old female mice $(n=24)$ were housed with a sexually experienced male for one night (the next day was designed as gestational day 0 ) and then placed in individual cages. The mice were randomly divided into control, stress, and stress with chewing groups ( $\mathrm{n}=8$ /group).

Mice were exposed to restraint stress for $45 \mathrm{~min}$, three times a day, from day 12 of pregnancy until delivery, by placing them in a ventilated plastic restraint tube (inner diameter: $4.5 \mathrm{~cm}$ ), in which they could move back and forth but not turn around, as previously reported $[9,10,20]$. Mice in the stress with chewing group were allowed to chew on a wooden stick (diameter: $2 \mathrm{~mm}$ ), which is made from virgin white birch tree, for $45 \mathrm{~min}$, three times a day during the stress period $[10,21]$. The wooden sticks were examined for signs of chewing at the end of the experimental period and all of them showed signs of chewing. All dams delivered their offspring vaginally. No differences in offspring birth weight were detected between groups. The offspring were raised by their biologic mothers until wearing. At wearing, male pups were selected from control, stress, and stress with chewing groups, assigned to control $(n=15)$, stress $(n=15)$, and stress with chewing $(n=15)$ groups, respectively, and housed 5 per group according to litter. Five-month-old male mice were used in the following experiments. Body weights of neonatal pups and offspring at 5 months of age were measured.

\section{Blood biochemical analysis}

At 5 months of age, all mice were administered by an overdose of sodium pentobarbital intraperitoneally. As the peak blood corticosterone level occurred at 20:00 [22], blood was sampled at 20:00 as described previously [22]. Animals were decapitated, blood was collected and centrifuged. The serum corticosterone level was measured by radioimmunoassay as reported previously $[9,10,21]$. The serum level of osteocalcin, a bone formation marker, was determined using a mouse osteocalcin enzyme-linked immunoassay kit (Biomedical Technologies, Stoughton, MA, USA). The serum level of N-telopeptide of type I collagen (NTx), a bone resorption marker, was measured using an enzyme-linked immunosorbent assay kit (TECOmedical, Sissach, Switzerland). 


\section{Micro-CT scanning and 3D microstructural analysis}

The 4th lumbar vertebrae (L4) and femurs were dissected and cleaned of adherent soft tissues. The bone samples were analyzed using cone-beam X-ray micro-CT system (MCTCB100MF, Hitachi Medical Corporation, Kashiwa, Japan) as described previously [23-25]. The L4 vertebral body and the distal femur were scanned with a tube voltage of $50 \mathrm{kV}$, tube current of $0.1 \mathrm{~mA}$. The voxel size was $10 \times 10 \times 10 \mu \mathrm{m}^{3}$. After micro-CT scanning, the image data was transferred to a workstation. The vertebral trabecular bone region was outlined for each micro-CT slice, excluding both the cranial and caudal endplate regions. Within these regions, trabecular bone was separated from cortical bone with boundaries defined by the endocortical bone surfaces. As for the distal femur, the region of interest was defined using 100 slices at approximately $0.5-2.0 \mathrm{~mm}$ away from the growth plate. The structural indices were calculated using a 3D trabecular bone analysis software TRI/3D-BON (Ratoc System Engineering Co. Ltd., Tokyo, Japan). TRI/3DBON builds 3D models from serial tomographic datasets for visualization and morphometric analysis as described $[9,10,25]$.

\section{Histomorphometric measurement}

Mice received calcein injections intraperitoneally (10 mg/ kg body weight) 8 and 2 days before sacrifice. After micro-CT scanning, both femurs and vertebrae were embedded in methyl methacrylate. The cross sections of vertebral body were cut at $150-\mu \mathrm{m}$ thickness using a low speed diamond saw (Isomet, Buehler, Lake Bluff, IL, USA), and then hand ground to a thickness of $20 \mu \mathrm{m}$ for dynamic bone histomorphometric analyses. The measurements included mineralizing surface (MS/BS), mineral apposition rate (MAR) and bone formation rate per bone surface (BFR/BS). For paraffin block preparations, the bone specimens were fixed in freshly prepared $4 \%$ paraformaldehyde, decalcified in $5 \%$ EDTA and embedded in paraffin by standard histologic procedures, as described previously $[9,10$, 26]. Then 5- $\mu \mathrm{m}$ sections were prepared for TRAP staining to evaluate osteoclast number (Oc.N/BS) and osteoclast surface (Oc.S/BS). All bone histomorphometric measurements were performed using Adobe Photoshop, as described previously [27].

\section{Statistical analysis}

All data are presented as means \pm SD. Statistical analysis was done using SPSS version 14 (Chicago, IL, USA). One-way analysis of variance (ANOVA) was used to compare the blood biochemical markers and the bone histomorphometric parameters in the control, stress, and stress with chewing groups. If the $\mathrm{F}$ test showed significance, Fisher's protected least significant differences test was used for post hoc multiple comparisons. Differences were considered statistically significant at $\mathrm{p}<0.05$.

\section{Results}

\section{The serum corticosterone levels in dams, and body weights and bone metabolic markers in offspring}

The serum corticosterone levels differed significantly between the control, stress, and stress with chewing groups in dams (Fig. 1a). As compared with the control group, the serum corticosterone level was significantly higher in the stress group. Chewing under chronic stress prevented the increase in the serum corticosterone level (Fig. 1a).

(A) Serum corticosterone level

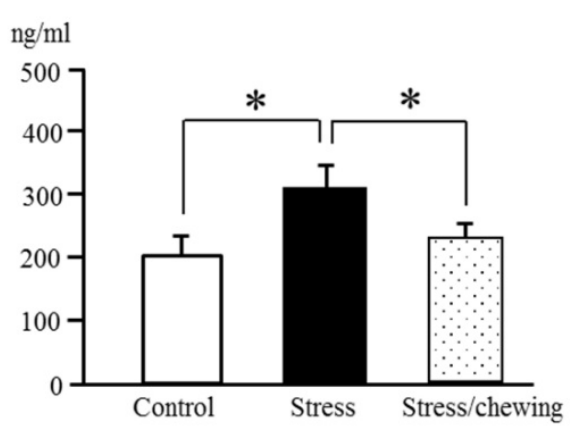

(B)

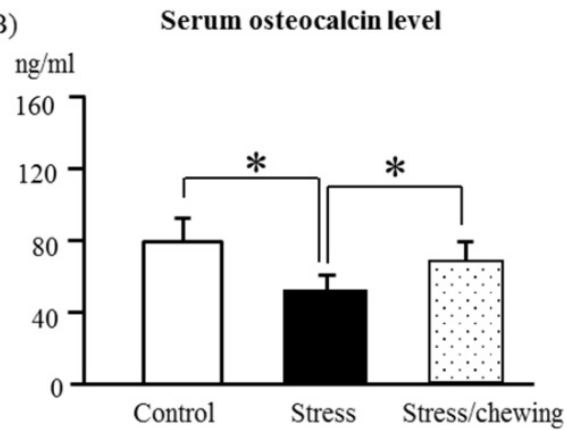

(C) Serum NTx level (nM BCE)

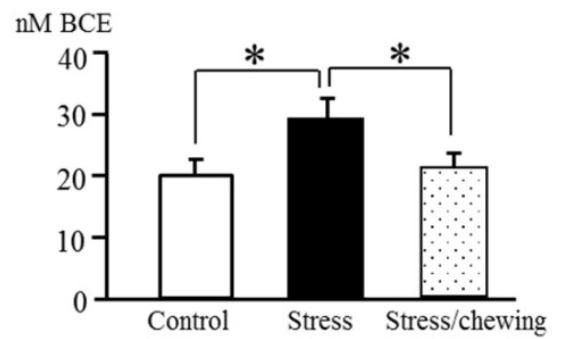

Figure 1. The serum corticosterone in dams (A), osteocalcin (B), and NTx (C) levels in offspring of the control, stress, and stress/chewing mice. All data represent as mean $\pm \mathrm{SD}$. ${ }^{\mathrm{p}} \mathrm{p}<0.05$. 
Body weights of neonatal pups ware similar in the control, stress, and stress/chewing groups. The mean weights of offspring at 5 months of age were $40.5 \mathrm{~g}, 38.8 \mathrm{~g}$, and $39.2 \mathrm{~g}$ in the control, stress, and stress/chewing groups, respectively. There was no significant difference in body weight changes among the three groups. The serum osteocalcin level in offspring was significantly decreased and the serum NTx level was significantly increased in the prenatal stress group (Fig. 1b, c). Compared with the stress group, the serum osteocalcin level was significantly higher and the serum NTx level was significantly lower in the stress with chewing group (Fig. 1b, c).

\section{Trabecular microstructural parameters in the vertebral body using micro-CT}

The microstructural parameters of the vertebral trabecular bone are shown in Fig. 2. As compared with the control group, trabecular bone volume fraction (BV/TV), trabecular thickness (Tb.Th), trabecular number (Tb.N) were significantly lower, and the trabecular separation (Tb.Sp) was significantly higher in the stress group. Compared to the stress group, BV/TV, Tb.Th and Tb.N were significantly higher, and Tb.Sp was significantly lower in the stress with chewing group (Fig. 2). We

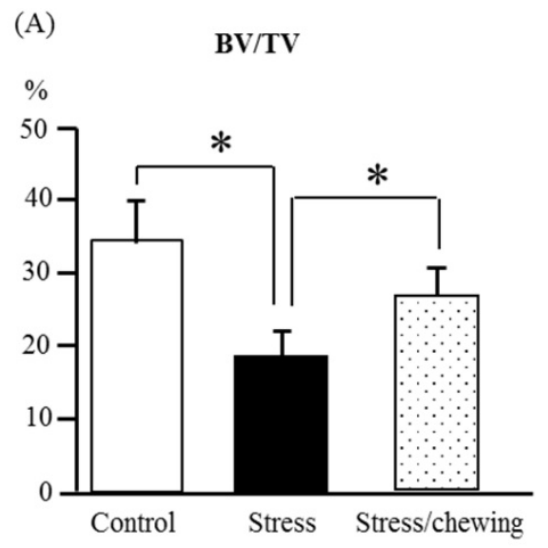

(B)

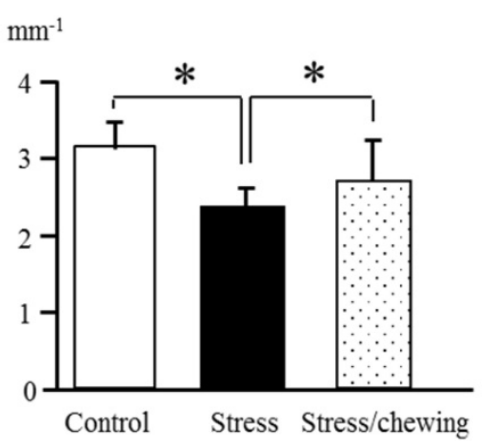

did not find any significant differences with regard to trabecular bone pattern factor and the degree of anisotropy among the three groups. Fig. 3 shows the typical 3D reconstructed micro-CT images of the L4 vertebral body. Images of the sample with $\mathrm{BV} / \mathrm{TV}$ that was closest to the mean $\mathrm{BV} / \mathrm{TV}$ were reconstructed in each group. There was a significant trabecular bone loss in the stress group compared to the control group. The trabecular volume was higher in the stress with chewing group than in the stress group (Fig. 3).

\section{Trabecular microstructural parameters in the distal femoral metaphysis using micro-CT}

The microstructural changes of the distal femoral trabecular bone are shown in Fig. 4. The change pattern for microstructural properties of the distal femoral trabecular bone resembled that of the vertebral trabecular bone. Compared with the control group, BV/TV, Tb.Th, and Tb.N were significantly lower, and Tb.Sp was significantly higher in the stress group. Compared with the stress group, BV/TV and Tb.N were significantly higher in the stress with chewing group. Tb.Th had a tendency to be higher and Tb.Sp had a tendency to be lower in the stress with chewing group (Fig. 4).

(C)

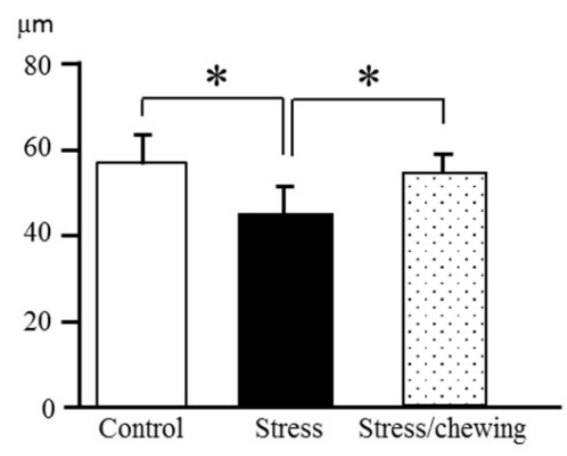

(D) Tb.Sp

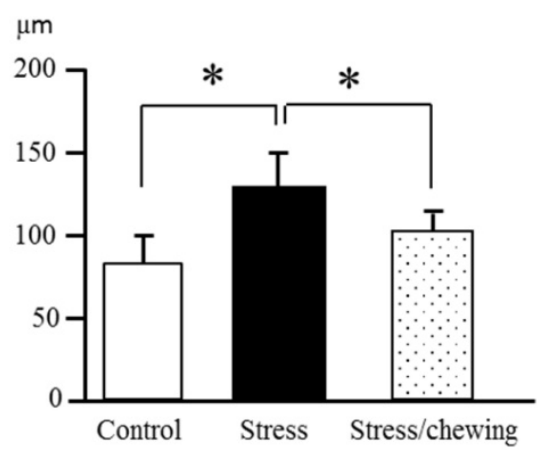

Figure 2. The microstructural properties of the vertebral trabecular bone. (A) Bone volume fraction (BV/TV), (B) Trabecular number (Tb.N), (C) Trabecular thickness (Tb.Th), and (D) Trabecular separation (Tb.Sp) in the control, stress, and stress/chewing mice. All data represent as mean \pm SD. *p $<0.05$. 

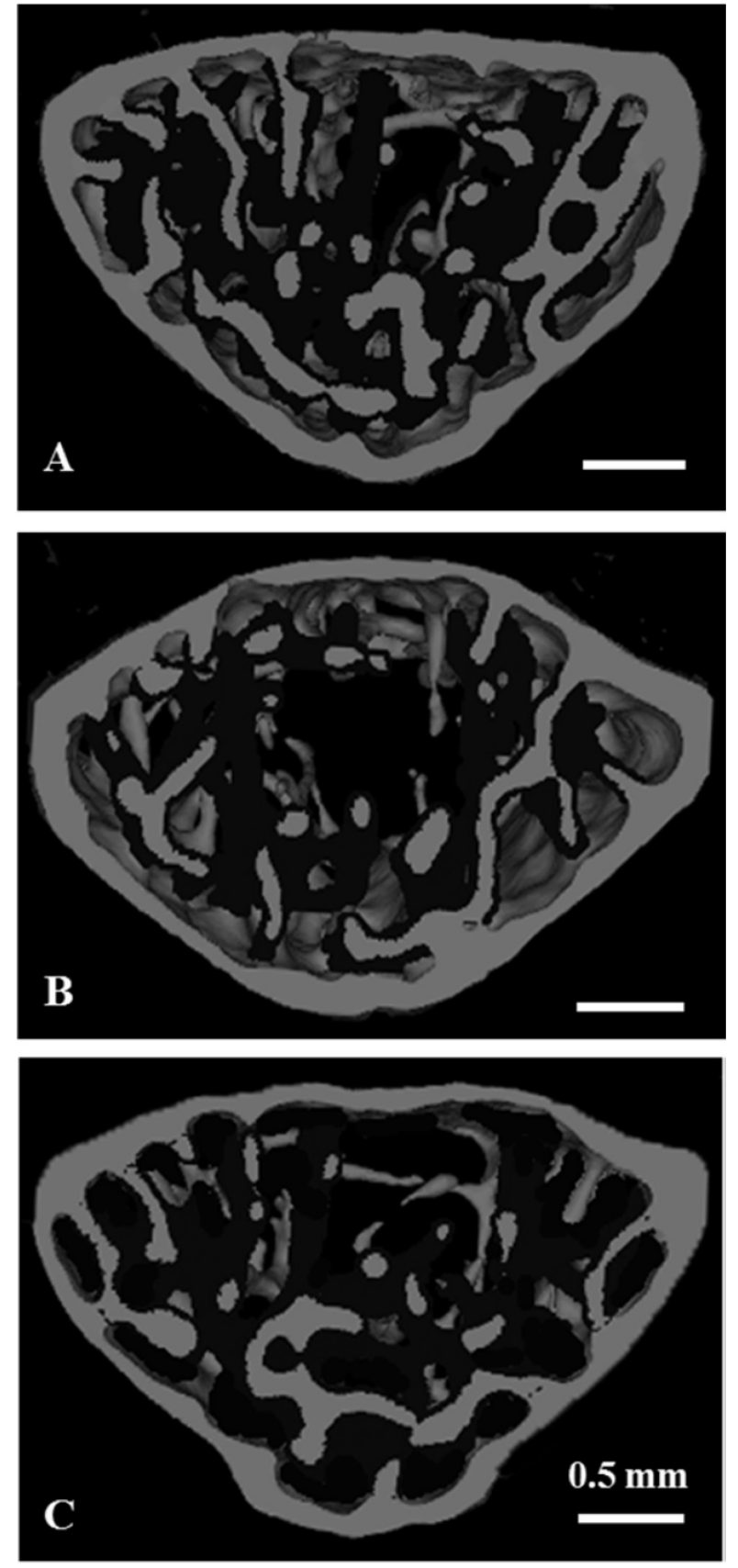

Figure 3. Three-dimensional micro-CT images representative the fourth lumbar vertebral body in the control (A), stress (B), and stress/chewing (C) mice. Images of the sample with the BV/TV that was closest to the mean BV/TV were presented in each group. Scale bar: $0.5 \mathrm{~mm}$.

\section{Bone histomorphometry}

The bone formation rate/bone surface (BFR/BS) in the stress group was significantly lower than that of the control group. Compared with the stress group, BFR/BS was significantly higher in the stress with chewing group (Fig. 5a). In the stress group, the osteoclast number (Oc.N/BS) and osteoclast surface (Oc.S/BS) were significantly higher compared the control group. Chewing under chronic stress suppressed the increases in Oc. N/BS and Oc.S/BS (Fig. 5b, c).

\section{Discussion}

In the present study, we investigated the effects of maternal active mastication during prenatal stress on chronic stress-induced lower bone mass and bone microstructural deterioration in mice using quantitative micro-CT, histomorphometry, and biochemical markers. We found that allowing dams to chew on a wooden stick during prenatal stress prevents the stress-induced increase in serum corticosterone levels in the dams. Prenatal stress induced a significant decrease of trabecular bone mass both in the vertebra and distal femur of the male adult offspring. Prenatal stress led to decrease of BV/TV by its effects to decrease Tb.Th, Tb.N, and increase Tb.Sp. The changes of trabecular histomorphometric parameters induced by prenatal stress are consistent with those observed in osteoporotic patients [23-25, $28,29]$.

Bone undergoes continuous remodeling, in which the old bone is resorbed by osteoclasts and new bone is formed by osteoblasts. Bone remodeling is strictly regulated by various factors. Previous studies showed that exposure to various stressors, such as restraint, immobilization, forced swimming, psychological, and social stressor lead to sustained increase in the circulating corticosterone level. Glucocorticoid-induced osteoporosis is one of the most common forms of secondary osteoporosis, accompanied by increased fracture risk. The detrimental effect of glucocorticoid excess on bone is caused by suppression of osteoblast bone formation and stimulation of osteoclast bone resorption [9-12, 30-32].

There is growing recognition that intrauterine environments may affect bone mineral accrual and thus the later osteoporosis risk [12, 14, 32, 33]. Bone size and bone mass increase from early embryogenesis through intrauterine, infant, childhood and adult to reach a peak. The peak bone mass achieved is a powerful determinant of later osteoporosis risk. Osteoporosis risk might be modified by intrauterine environments. Maternal exposure to various psychological stresses during pregnancy results in abnormalities in offspring in various tissues, including bone, and elevated maternal glucocorticoids likely play a key role in mediating these effects $[14,32]$. The placenta serves as a critical interface between maternal and fetal physiology and forms a protective barrier to maternal glucocorticoids [34]. Chronic psychological prenatal stress impairs the placental function, resulting in elevation of the fetal glucocorticoid level, which is 
directly linking programming effects and increases osteoporosis risk in adult offspring [35]. Some studies indicated that prenatal stress is capable of producing a reduction in birth weight, suggesting intrauterine growth retardation [36]. While others reported that there were no weight changes in prenatal stress offspring [37]. The present studies showed that there was no significant difference in body weight changes among the control, stress, and stress/chewing groups. The difference in the stress protocol and the timing of stress could explain the disparity in findings between studies. The detail mechanism by which prenatal stress cause intrauterine growth retardation needs further investigation.

Recent studies indicated that chewing during restraint stress suppressed the stress-induced increase in circulating glucocorticoid levels and noradrenaline turnover in the hypothalamus and limbic system [12, $18,21]$. Chewing is an effective stress-coping behavior. Chewing during stress could attenuate the stress-induced elevation in corticosterone levels and noradrenaline turnover. Animals provided the opportunity to chew during restraint stress exhibited

(A)

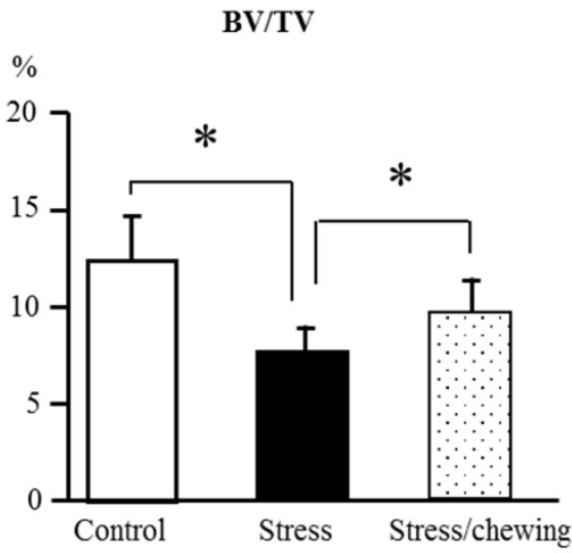

(B)

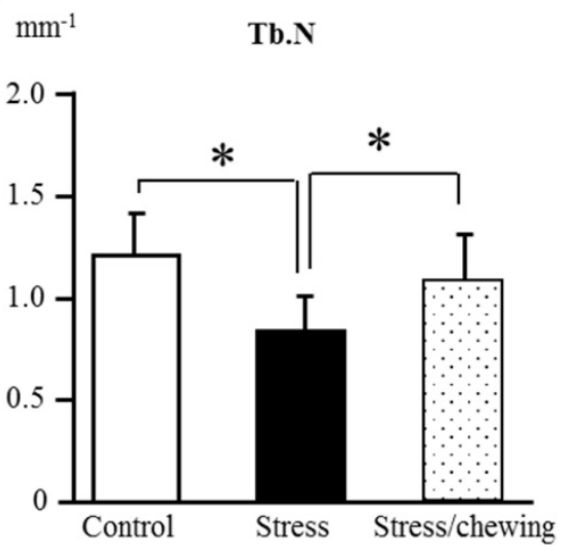

decreases in stress-induced corticosterone levels and attenuated HPA axis and autonomic nervous system responses to stress, which helps to prevent the prenatal stress-induced development of osteoporosis $[11,18]$. Maternal chewing during exposure to prenatal stress attenuated the increase in prenatal stress-induced corticosterone levels. Furthermore, adult offspring of dams exposed to prenatal stress exhibited impaired bone remodeling in offspring, which was attenuated by allowing the dams to chew on a wooden stick during exposure to prenatal stress. Therefore, maternal chewing during prenatal stress appears to be effective for preventing lower bona mass in the adult offspring.

The findings of the present study indicate that active mastication during prenatal stress in dams could effectively ameliorate prenatal stress-induced lower trabecular bone volume of the vertebra and femur in the adult offspring. Active mastication during prenatal stress may be an effective strategy for the dams to prevent prenatal stress-related lower bone mass in the offspring.

(C)

Tb.Th

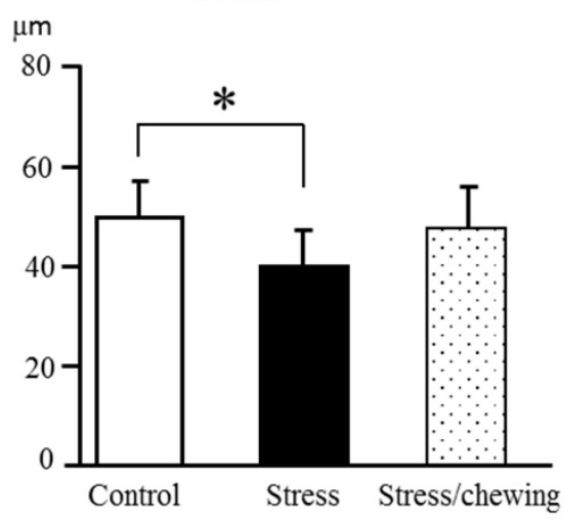

(D)

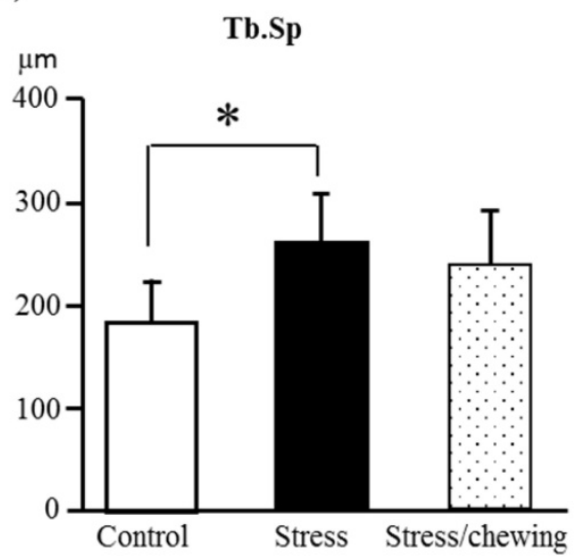

Figure 4. The microstructural properties of the distal femoral metaphyseal trabecular bone. (A) Bone volume fraction (BV/TV), (B) Trabecular number (Tb.N), (C) Trabecular thickness (Tb.Th), and (D) Trabecular separation (Tb.Sp) in the control, stress, and stress/chewing mice. All data represent as mean \pm SD. *P $<0.05$. 
(A)

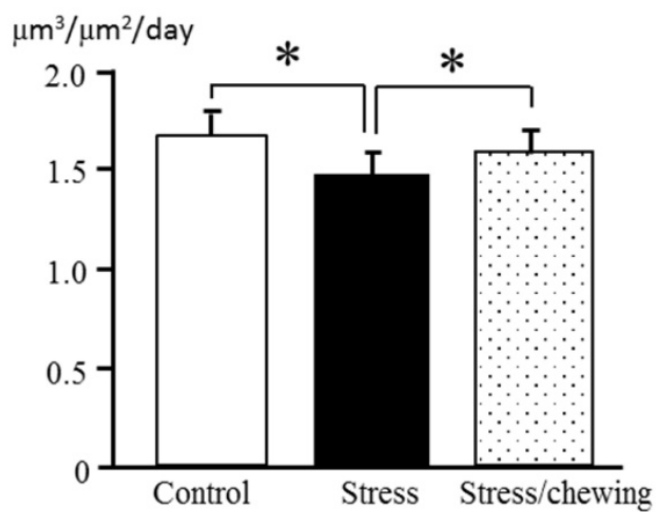

(B)

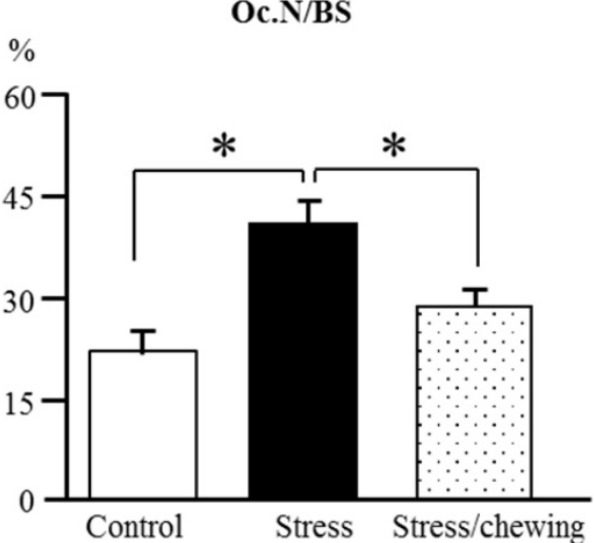

(C) $\mathrm{mm}^{-1}$

Oc.S/BS)

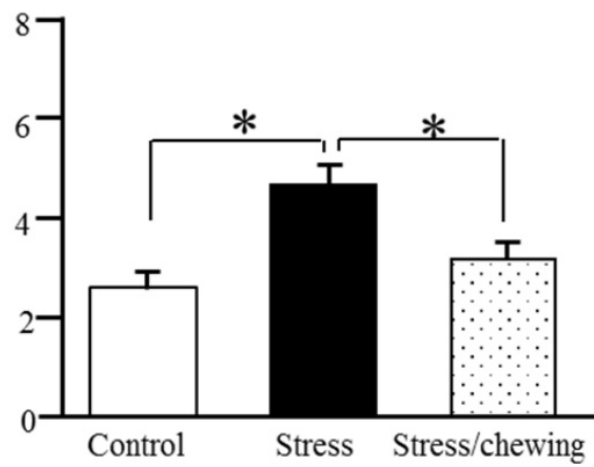

Figure 5. The bone remodeling parameters in the control, stress, and stress/chewing mice. (A) The bone formation rate (BFR/BS), (B) The osteoclast number (Oc.N/BS), and (C) The osteoclast surface (Oc.S/BS. All data represent as mean $\pm \mathrm{SD}$. ${ }^{*} \mathrm{p}<0.05$.

\section{Acknowledgements}

This work was supported in part by a Grant-in-Aid for Scientific Research (KAKENHI 22390395, 26462916, 15K15761) from the Japan Society for the Promotion of Science.

\section{Competing Interests}

The authors have declared that no competing interest exists.

\section{References}

1. Rachner TD, Khosla S, Hofbauer LC. Osteoporosis: now and the future. Lancet. 2011; 377:1276-1287.

2. Chen H, Zhou X, Fujita H, Onozuka M, Kubo KY. Age-related changes in trabecular and cortical bone microstructure. Int J Endocrinol. 2013; doi:10.1155/2013/213234.

3. Chen H, Hayashi T, Zhou X, et al. Sophisticated imaging technology in the assessment of osteoporosis risk. In: Dionyssiotis Y, ed. Osteoporosis. Rijeka: Intech; 2013: 181-194

4. National Institutes of Health Office of the Direction. Osteoporosis prevention, diagnosis, and therapy. NIH Consensus Statement. 2000; 17:1-45.

5. Proietti R, Mapelli D, Volpe B, et al. Mental stress and ischemic heart disease: evolving awareness of a complex association. Future Cardiol. 2011; 7:425-437.

6. Marin MF, Lord C, Andrews J, et al. Chronic stress, cognitive functioning and mental health. Neurobiol Learn Mem. 2011; 96:583-595.

7. Yirmiya R, Goshen I, Bajayo A, et al. Depression induces bone loss through stimulation of the sympathetic nervous system. Proc Natl Acad Sci USA. 2006; 103:16876-16881.

8. Cizza G, Primma S, Csako G. Depression as a risk factor for osteoporosis. Trends Endocrinol Metab. 2009; 20:367-373.

9. Furuzawa M, Chen H, Fujiwara S, et al. Chewing ameliorates chronic mild stress-induced bone loss in senescence-accelerated mouse (SAMP8), a murine model of senile osteoporosis. Exp Gerontol. 2014; 55:12-18.

10. Azuma K, Furuzawa M, Fujiwara S, et al. Effects of Active mastication on chronic stress-induced bone loss in mice. Int J Med Sci. 2015; 12:952-957.

11. Azuma K, Adachi Y, Hayashi H, Kubo KY. Chronic psychological stress as a risk factor of osteoporosis. J UOEH. 2015; 37:245-253.

12. Chen $H$, Iinuma $M$, Onozuka $M$, Kubo KY. Chewing maintains hippocampus-dependent cognitive function. Int J Med Sci. 2015; 12:502-509.

13. Harvey N, Dennison E, Cooper C. Osteoporosis: a lifecourse approach. J Bone Miner Res. 2014; 29:1917-1925.

14. Dennison EM, Harvey NC, Cooper C. Programming of osteoporosis and impact on osteoporosis risk. Clin Obstet Gynecol. 2013; 56:549-555.

15. Heindel JJ, Vandenberg LN. Developmental origins of health and disease: a paradigm for understanding disease etiology and prevention. Curr Opin Pediatr. 2015; 27:248-253.

16. Entringer S, Buss C, Wadhwa PD. Prenatal stress, development, health and disease risk: A psychobiological perspective-2015 Curt Richter Award Paper. Psychoneuroendocrinology. 2015; 62:366-375.

17. Kubo KY, Chen H, Onozuka M. The relationship between mastication and cognition. In: Wang $\mathrm{Z}$, Inuzuka $\mathrm{H}$, eds. Senescence and senescence-related disorders. Rijeka: Intech; 2013: 115-132.

18. Kubo KY, linuma M, Chen H. Mastication as a stress-coping behavior. Biomed Res Int. 2015; doi:10.1155/2015/876409.

19. Curtis EM, Moon RJ, Dennison EM, Harvey NC. Prenatal calcium and vitamin D intake, and bone mass in later life. Curr Osteoporos Rep. 2014; 12:194-204.

20. Kubo KY, Sasaguri K, Ono Y, et al. Chewing under restraint stress inhibits the stress-induced suppression of cell birth in the dentate gyrus of aged SAMP8 mice. Neurosci Lett. 2009; 466:109-113.

21. Onishi M, Iinuma M, Tamura Y, Kubo KY. Learning deficits and suppression of the cell proliferation in the hippocampal dentate gyrus of offspring are attenuated by maternal chewing during prenatal stress. Neurosci Lett. 2014; 560:77-80.

22. Onozuka M, Watanabe K, Fujita M, et al. Evidence for involvement of glucocorticoid response in the hippocampal changes in aged molarless SAMP8 mice. Behav Brain Res. 2002; 131:125-129.

23. Chen $\mathrm{H}$, Shoumura S, Emura S, Bunai Y. Regional variations of vertebral trabecular bone microstructure with age and gender. Osteoporos Int. 2008; 19:1473-1483.

24. Chen H, Zhou X, Shoumura S, Emura S, Bunai Y. Age- and gender-dependent changes in three-dimensional microstructure of cortical and trabecular bone at the human femoral neck. Osteoporos Int. 2010; 21:627-636.

25. Chen H, Kubo KY. Segmental variations in trabecular bone density and microstructure of the spine in senescence-accelerated mouse (SAMP6): a murine model for senile osteoporosis. Exp Gerontol. 2012; 47:317-322.

26. Feng JQ, Zhang J, Dallas SL, et al. Dentin matrix protein 1, a target molecule for Cbfa1 in bone, is a unique bone marker gene. J Bone Miner Res. 2002; 17:1822-1831.

27. Egan KP, Brennan TA, Pignolo RJ. Bone histomorphometry using free and commonly available software. Histopathology. 2012; 61:1168-1173.

28. Riggs BL, Parfitt AM. Drugs used to treat osteoporosis: the critical need for a uniform nomenclature based on their action on bone remodeling. J Bone Miner Res. 2005; 20:177-184.

29. Chen H, Zhou X, Washimi Y, Shoumura S. Three-dimensional microstructure of the bone in a hamster model of senile osteoporosis. Bone. 2008; 43:494-500.

30. Compston J. Management of glucocorticoid-induced osteoporosis. Nat Rev Rheumacol. 2010; 6:82-88. 
31. Weinstein RS. Clinical practice. Glucocorticoid-induced bone disease. N Engl J Med. 2011; 365:62-70.

32. Holroyd C, Harvey N, Dennison E, Cooper C. Epigenetic influences in the developmental origins. Osteoporos Int. 2012; 23:401-410.

33. Curtis EM, Moon RJ, Dennison EM, Harvey NC. Prenatal calcium and vitamin D intake, and bone mass in later life. Curr Osteoporos Rep. 2014; 12:194-204.

34. Togher KL, O'Keeffe MM, Khashan AS, et al. Epigenetic regulation of the placental HSD11B2 barrier and its role as a critical regulator of fetal development. Epigenetics. 2014; 9:816-822.

35. Jensen Peña C, Monk C, Champagne FA. Epigenetic effects of prenatal stress on 11 $\beta$-hydroxysteroid dehydrogenase- 2 in the placenta and fetal brain. PLoS One. 2012; 7:e39791.

36. Lesage J, Del-Favero F, Leonhardt M, et al. Prenatal stress induces intrauterine growth restriction and programmes glucose intolerance and feeding behaviour disturbances in the aged rat. J Endocrinol. 2004; 181:291-296.

37. D'mello AP, Liu Y. Effects of maternal immobilization stress on birth weight and glucose homeostasis in the offspring. Psychoneuroendocrinology. 2006; 31:395-406. 